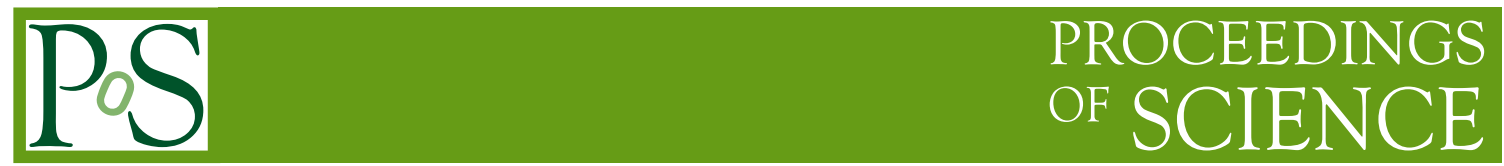

\title{
Hadron physics at KLOE and KLOE-2
}

\section{Paolo GAUZZI *}

Dipartimento di Fisica, Sapienza Università di Roma e INFN Sezione di Roma, P.le A.Moro 2, 00185 Rome (Italy)

E-mail: Paolo.Gauzzi@romal.infn.it

\begin{abstract}
The most recent results from the KLOE experiment on hadron physics are presented. $\eta$ mesons produced in the radiative decay $\phi \rightarrow \eta \gamma$ have been used to measure the rare decays $\eta \rightarrow \pi^{+} \pi^{-} \gamma$ and $\eta \rightarrow e^{+} e^{-} e^{+} e^{-}$. The decay $\phi \rightarrow \eta e^{+} e^{-}$has been used to search for light dark vector mesons. $\gamma \gamma$ collisions have also been exploited to study the single $\eta$ production, and the $\pi^{0} \pi^{0}$ final state looking for the contribution of the scalar $\sigma(600)$.

Some prospects for the new data-taking of KLOE starting at the beginning of 2012 at the upgraded DA $\Phi$ NE are also discussed.
\end{abstract}

The 2011 Europhysics Conference on High Energy Physics-HEP 2011,

July 21-27, 2011

Grenoble, Rhône-Alpes France

\section{${ }^{*}$ on behalf of the KLOE/KLOE-2 Collaboration}



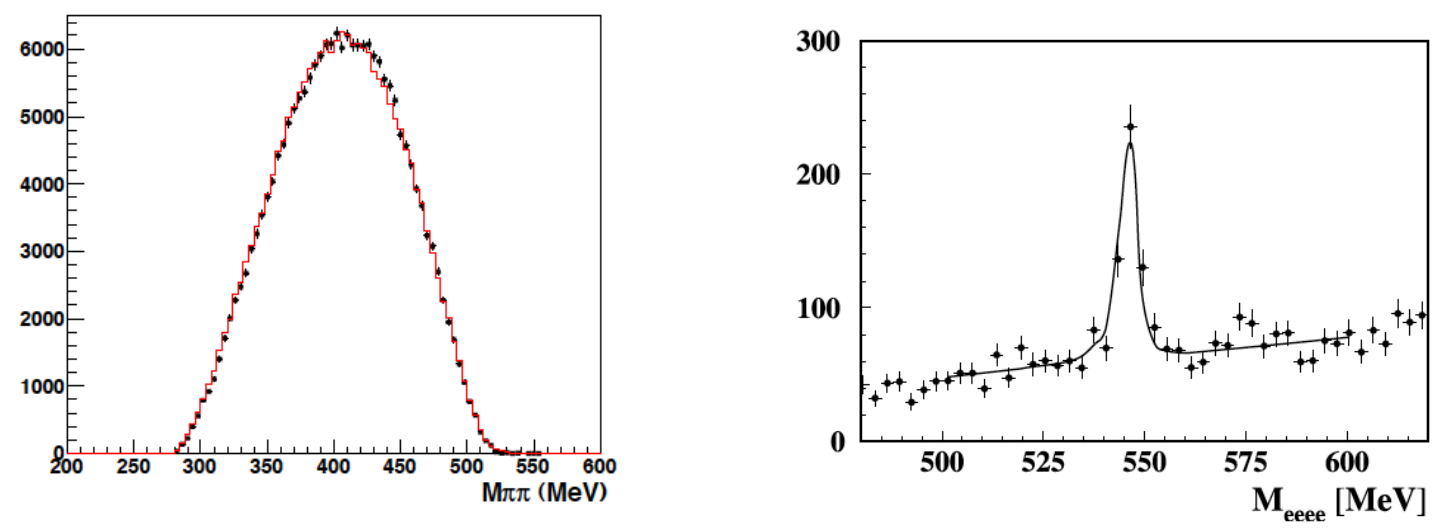

Figure 1: Left - Two pion invariant mass distribution for $\eta \rightarrow \pi^{+} \pi^{-} \gamma$, points: data; histogram: fit function. Right - Invariant mass distribution of $\eta \rightarrow e^{+} e^{-} e^{+} e^{-}$.

Introduction. From 2000 to $2006 \mathrm{KLOE}$ has collected $2.5 \mathrm{fb}^{-1}$ of data at the peak of the $\phi(1020)$ plus $250 \mathrm{pb}^{-1}$ off-peak at the $e^{+} e^{-}$collider DA $\Phi$ NE in Frascati. In this paper the recent KLOE results on hadron physics are reported. During 2008 a new interaction scheme of the DAФNE $\phi$-factory has been succesfully tested, reaching a peak luminosity of about a factor of three larger than what previously obtained. Following these achievement, a new data-taking with an improved detector will start at the beginning of 2012 (KLOE-2 experiment). The KLOE-2 present upgrade consists in the installation of two different $e^{ \pm}$taggers for $\gamma \gamma$ physics: the Low Energy Tagger, made of two crystal calorimeters placed very near the DA $\Phi$ NE Interaction Point (IP), and the High Energy Tagger, made of two position sensitive detectors placed far from the IP, after the first bending dipoles of DA $\Phi N E$. After the collection of about $5 \mathrm{fb}^{-1}$ in one year, a major detector upgrade is planned, aiming to collect about $20 \mathrm{fb}^{-1}$ of data to complete the KLOE physics program $[1]$.

Rare $\eta$ decays. The properties of the $\eta$ meson can be studied through the radiative decay $\phi \rightarrow$ $\eta \gamma$. The full KLOE data set corresponds to about $10^{8} \eta$ produced. The process $\eta \rightarrow \pi^{+} \pi^{-} \gamma$ is described by the so called box anomaly, which is a higher order term of the Wess-Zumino-Witten Lagrangian. From the experimental point of view it is relevant to check if a contact term is needed besides the resonant contribution, dominated by the $\rho$ meson. Both the branching ratio and the two pion invariant mass distribution are sensitive to the contact term [2]. The previous measurements date back to the '70's, the most recent result by CLEO shows a two $\sigma$ discrepancy with the older ones. From a sample of $558 \mathrm{pb}^{-1}$, the measurement of the branching ratio normalized to $\eta \rightarrow \pi^{+} \pi^{-} \pi^{0}$ has been obtained, $\Gamma\left(\eta \rightarrow \pi^{+} \pi^{-} \gamma\right) / \Gamma\left(\eta \rightarrow \pi^{+} \pi^{-} \pi^{0}\right)=0.1838 \pm 0.0005 \pm 0.0030$ in good agreement with the CLEO result. The normalization sample is clean and well kept under control as is demonstrated by the evaluation of its branching ratio: we selected about $1.2 \times 10^{6}$ events from which we obtain $\operatorname{Br}\left(\eta \rightarrow \pi^{+} \pi^{-} \pi^{0}\right)=(22.41 \pm 0.03 \pm 0.35) \%$ to be compared with the PDG value $(22.74 \pm 0.28) \%$. In fig.1(left) is shown a fit to the $M_{\pi \pi}$ distribution, after the background subtraction, with the parametrization from ref.[3]. The $\pi^{+} \pi^{-}$lineshape of the $\eta^{\prime} \rightarrow \pi^{+} \pi^{-} \gamma$ is more sensitive to the contact term; in the first year of KLOE- 2 we expect to collect $10^{5}$ events of such process. The decay $\eta \rightarrow e^{+} e^{-} e^{+} e^{-}$proceeds through two virtual photons intermediate state, 

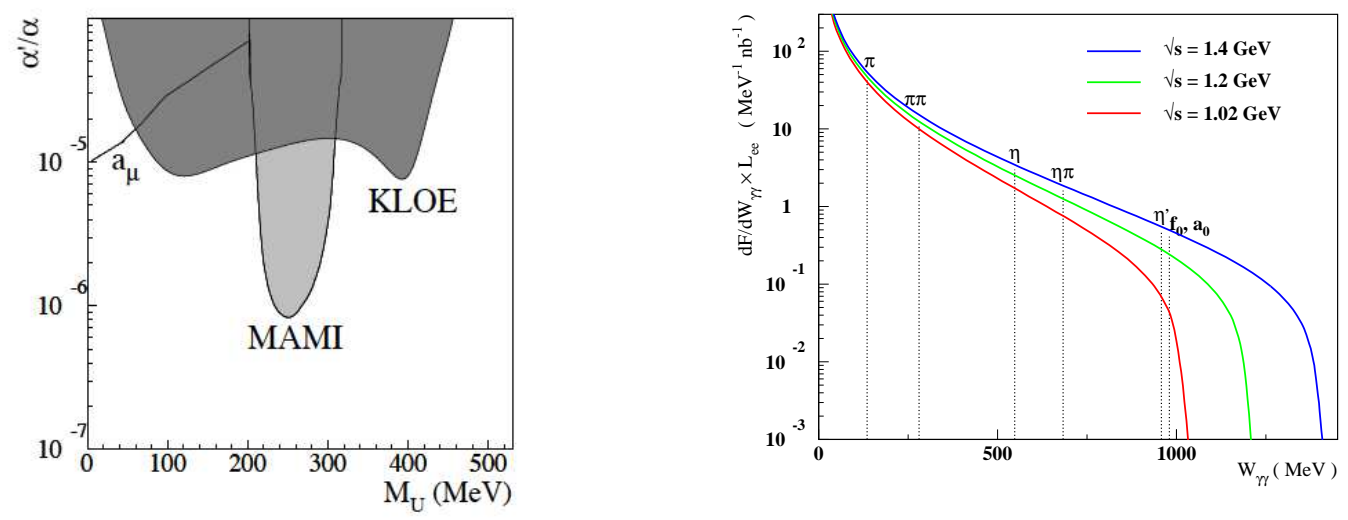

Figure 2: Left - Exclusion plot at 90\% C.L. for $\alpha^{\prime} / \alpha$ compared with the existing limits in our region of interest. Right - Differential photon-photon flux function for different center of mass energies.

with photon conversion to $e^{+} e^{-}$. It is interesting to study the $\eta$ transition form factor for timelike $q^{2}$ of the photons. The theoretical expectation for the branching ratio is around $2.5 \times 10^{-5}$. Two upper limits at $90 \%$ C.L., based on few events, have been set by the CMD-2 Collaboration, $\operatorname{Br}\left(\eta \rightarrow e^{+} e^{-} e^{+} e^{-}\right)<6.9 \times 10^{-5}$, and by WASA at CELSIUS, $\operatorname{Br}\left(\eta \rightarrow e^{+} e^{-} e^{+} e^{-}\right)<9.7 \times 10^{-5}$. From the analysis of $1.7 \mathrm{fb}^{-1}$ we obtain the first evidence of this decay. In fig.1(right) the four lepton invariant mass of the final sample is reported: we evaluate $362 \pm 29$ signal events that correspond to $\operatorname{Br}\left(\eta \rightarrow e^{+} e^{-} e^{+} e^{-}\right)=(2.4 \pm 0.2 \pm 0.1) \times 10^{-5}[4]$.

Search for a light dark gauge boson. An explanation of recent astrophysical observations based on the existence of a dark sector that interacts with the Standard Model (SM) particles through the mixing of a new light gauge boson, $U$ of $O(1 \mathrm{GeV})$ mass, with the SM hypercharge gauge field has been proposed [5]. The existence of such a new particle has been tested by using the decay chain $\phi \rightarrow \eta U, U \rightarrow e^{+} e^{-}$, selecting the decay $\eta \rightarrow \pi^{+} \pi^{-} \pi^{0}$. The irreducible background is the Dalitz decay $\phi \rightarrow \eta e^{+} e^{-}$. No evidence has been found in $1.5 \mathrm{fb}^{-1}$ of data. The exclusion plot for the ratio of the $U$-boson coupling to the electric charge and the fine structure constant is shown in fig. $2, \alpha^{\prime} / \alpha<2 \times 10^{-5}$ at $90 \%$ C.L. in the range $50<M_{U}<420 \mathrm{MeV}$ [6].

$\gamma \gamma$ physics. In $\gamma \gamma$ processes, like $e^{+} e^{-} \rightarrow e^{+} e^{-} \gamma^{*} \gamma^{*} \rightarrow e^{+} e^{-} X, C=+1$ hadronic states can be produced. If both photons are quasi-real, the event yield evaluated in the Equivalent Photon Approximation is $N_{e e X}=L \int \frac{d F}{d W_{\gamma \gamma}} \sigma_{\gamma \gamma \rightarrow X}\left(W_{\gamma \gamma}\right) d W_{\gamma \gamma}$, where $W_{\gamma \gamma}$ is the $\gamma \gamma$ invariant mass, $L$ is the integrated luminosity and $d F / d W_{\gamma \gamma}$ is the flux function (fig.2). At the DAФNE energy the final states with a single $\pi^{0}$ or $\eta$ as well as the $\pi \pi$ one can be detected. The latter is interesting for the study of the $\sigma(600)$, via the reaction $\gamma \gamma \rightarrow \sigma(600) \rightarrow \pi \pi$. Since KLOE took data without taggers for scattered leptons the off-peak sample, $250 \mathrm{pb}^{-1}$ collected $\sqrt{s}=1 \mathrm{GeV}$ has been exploited to avoid the large background from the $\phi$. The cleanest channel is $\gamma \gamma \rightarrow \pi^{0} \pi^{0}$; events with only four prompt photons have been selected, the scattered leptons are not detected as they escape in the beam-pipe. In fig. 3 the distribution of the four photon invariant mass is shown; the background has been evaluated according to the expected cross-sections of the various processes. Work is in progress to extract $\sigma\left(\gamma \gamma \rightarrow \pi^{0} \pi^{0}\right)$. In the same data sample the $\eta$ meson production has been also 

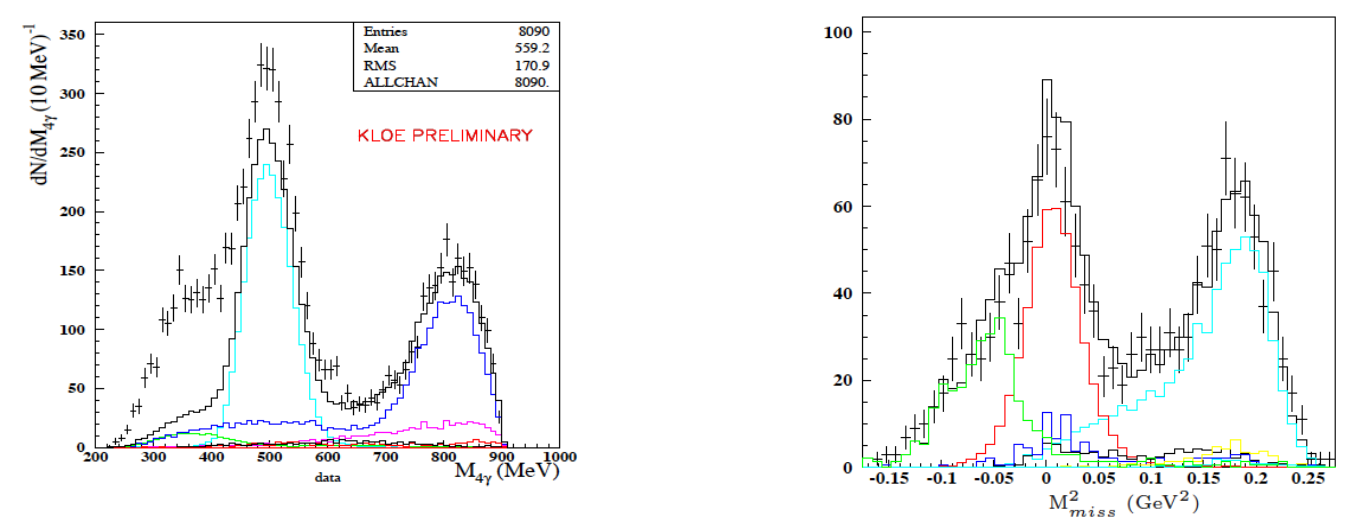

Figure 3: Left $-e^{+} e^{-} \rightarrow e^{+} e^{-} \pi^{0} \pi^{0}$, four photon invariant mass: points $=$ data, histograms $=$ background from MC. Right - Missing mass for $e^{+} e^{-} \rightarrow e^{+} e^{-} \eta$ : points $=$ data, light blue histogram $=$ signal MC.

studied by selecting $\eta \rightarrow \pi^{+} \pi^{-} \pi^{0}$ and $\eta \rightarrow \pi^{0} \pi^{0} \pi^{0}$. In fig.3 the missing mass for the charged channel is reported. After the background subtraction 650 signal events have been found; the corresponding missing mass distribution is shown in fig.3. In the neutral channel 921 signal events have been obtained. Work is in progress to exract $\sigma\left(e^{+} e^{-} \rightarrow e^{+} e^{-} \eta\right)$. As a by-product we measured the cross-section of the main background process for the two photon production of $\eta$ mesons, $\sigma\left(e^{+} e^{-} \rightarrow \eta \gamma, \sqrt{s}=1 \mathrm{GeV}\right)==(0.866 \pm 0.009 \pm 0.093) \mathrm{nb}$. The KLOE-2 data-taking will be mostly at the peak of the $\phi$, then the taggers will be essential to suppress the background and to close the kinematics of the events by detecting the scattered leptons. A precision measurement of $\sigma\left(\gamma \gamma \rightarrow \pi^{0} \pi^{0}\right)$, to improve the present experimental knowledge in the region of $W_{\gamma \gamma}<800 \mathrm{MeV}$, is planned [1]. Concerning single pseudoscalar final states, the two photon decay width of $\pi^{0}$ and $\eta$ can be measured, as well as the transition form factor, $F_{\pi^{0} \gamma^{*} \gamma^{*}}\left(q_{1}^{2}, q_{2}^{2}\right)$, relevant for the calculation of the hadronic light-by-light scattering contribution to $g-2$ of the muon.

Conclusions. KLOE has given a relevant contribution to hadron physics with the $2.5 \mathrm{pb}^{-1}$ collected during the 2001-2006 data-taking. In 2008 has been shown that with a new interaction scheme DA $\Phi N E$ could increase the luminosity by a factor of about three. A new data-taking campaign is now starting with the KLOE detector upgraded, at first with taggers for $\gamma \gamma$ physics, and then with an Inner Tracker and new small angle calorimeters. We plan to collect about 20 $\mathrm{pb}^{-1}$ in the next three years, improving the precision on many of the performed measurements and studying new final states.

\section{References}

[1] G.Amelino-Camelia et al., Eur.Phys.J.C68 (2010) 619.

[2] M.Benayoun et al., Eur.Phys.J.C31 (2003) 525.

[3] C.Picciotto, Phys.Rev. D45 (1992) 1569.

[4] KLOE Collaboration, F.Ambrosino et al., Phys.Lett. B702 (2011) 324.

[5] N.Arkani-Hamed et al., Phys.Rev. D79 (2009) 015014.

[6] KLOE-2 Collaboration, F.Archilli et al., Phys.Lett. B706 (2012), 251. 